\title{
The Pattern Recognition Technology Application in the Electronic Information Feature Extraction
}

\author{
Ruijuan Du, Shilin Cui \\ Nanyang Institute of Technology, China
}

Keywords: Pattern recognition, application, electronic information, feature extraction

\begin{abstract}
Through investigation and research on the development and status quo of pattern recognition, it is understood that the theory and methods of pattern recognition have been widely used in many fields of science and technology, which has greatly promoted the development of artificial intelligence systems and expanded the characteristics of electronic information. The research of pattern recognition mainly focuses on how the objects of the graduate students perceive objects, and how to use computer to realize pattern recognition theory and methods under a given task. This paper describes in detail the application of pattern recognition technology in electronic information feature extraction.
\end{abstract}

\section{Introduction}

Pattern recognition was born in the 1920s. With the advent of computers in the 1940s and the rise of artificial intelligence in the 50s, pattern recognition rapidly developed into a discipline in the early 60s. What is pattern and pattern recognition? Broadly speaking, things that are observable in time and space can be called patterns if they can be distinguished whether they are the same or similar. In a narrow sense, patterns are time-produced by observing specific individual things [1]. The information of the spatial distribution is the class to which the pattern belongs or the pattern in the same class is called a pattern class (or simply a class). The "pattern recognition" is based on certain measurements or observations to classify the patterns to be recognized into their respective pattern classes.

After years of research and development, pattern recognition technology has been widely used in artificial intelligence, computer engineering, robotics, neurobiology, medicine, detective science, and high-energy physics, archeology, geological exploration, aerospace science, and weapon technology. Fields, such as speech recognition, speech translation, face recognition, fingerprint recognition, biometric authentication technology, etc. The importance of pattern recognition technology for the development of national economy and the development of national defense science and technology has been recognized and widely valued by people [1]. This article will introduce in detail the basic issues involved in pattern recognition, the research field and the current status of the current development, and look forward to the development trend of pattern recognition.

\section{The basic theory of pattern recognition technology}

Pattern recognition is the basis of artificial intelligence. With the development of computer and artificial intelligence technology, pattern recognition has become more and more widely used in image processing. In recent years, pattern recognition has also made many impressive achievements. There are many non-negligible progresses. Digital image processing, also known as computer image processing, refers to the process of converting an image signal into a digital signal and processing it with a computer [2]. The image is the main source of human acquisition and exchange of information. The application of image processing must involve all aspects of human life and work. With the continuous expansion of human activities, the application areas of image processing will also continue to expand. Image recognition based on pattern recognition has become the direction of image research in the field of image recognition with the development of computer and artificial intelligence technology [2]. This paper first introduces the basic theory and basic methods of image pattern recognition, then explains the application theory of pattern recognition in image processing, 
and finally exemplifies the specific application of pattern recognition in image processing.

Pattern recognition is a sorting process by which the computer processes and discriminates information. It is an important branch of signal processing and artificial intelligence. Artificial intelligence is a discipline that specializes in using robots to imitate people's movements, feelings, and thinking processes and laws. Pattern recognition is the use of computer-based mathematical techniques to study the automatic processing and interpretation of patterns. We call the environment and the object collectively the "model." With the development of computer technology and artificial intelligence, humans may study complex information processing processes [1]. An important form of information processing is the identification of the environment and objects by living bodies. Of particular importance to humans is the identification of optical information (obtained by visual organs) and acoustic information (obtained by auditory organs). This is two important aspects of pattern recognition.

\section{Image Processing Based on Pattern Recognition Technology}

\subsection{Image Segmentation Based on Pattern Recognition Technology}

The technique and process of dividing an image into its characteristic regions by relevance and extracting the desired objects is called image segmentation. The key to segmentation lies in the determination of the segmentation basis. From the perspective of pattern recognition technology, the image segmentation problem is considered. Segmentation is an object that needs to be segmented according to the image. According to the structural characteristics of the image, all the components of the image are divided into two categories: "split" and "non-split". For any one thing has some essential characteristics that are different from other things, it can be extracted that the essential features can be distinguished from the segmented background image and used as the basis for identifying things [2]. When segmenting an image positioning object, a feature space composed of features may be selected for position recognition. Therefore, the segmentation object is regarded as the object of pattern recognition. The process of image segmentation is to find a specific pattern class in pattern recognition, and according to the characteristics of the pattern class, combined with its corresponding segmentation technique.

Image recognition is an advanced stage of image processing. Its research is to analyze and identify the visual images of surrounding objects through the instrument, so that effective conclusion can be obtained. However, in order for computer systems to be able to recognize images recognized by the human visual system as well, one must develop computational methods and analyze image features, so that pattern recognition techniques are applied to image recognition, and then image features can be mathematically represented and taught. Computers can also recognize and identify these features [2].

In pattern recognition, some pattern classification techniques have been used. Some of these technologies are called decision theory techniques, in which the unknown pattern classification is determined by the basic principles of certain deterministic, statistical, or fuzzy theories.

Decision theory pattern recognition techniques are mainly divided into classification methods based on supervised learning and classification methods using unsupervised techniques.

Supervised classification methods can be further divided into parameter classifiers and nonparametric classifiers. In parametrically supervised classification, a large number of labeled training sample pattern sets are used to train the classifier and the statistical parameters of each type of model are estimated [3]. Among them, the minimum distance classifier and the maximum likelihood classifier are frequently used supervised algorithms.

Unsupervised classification technology does not consider parameters, and often uses some non-parametric techniques such as K nearest neighbor technology, Parzen window technology, and the like. In the unsupervised case, the entire dataset is partitioned according to some similar standard machines, and the structure generates a cluster set. Each cluster set in the pattern belongs to a specific class. 


\subsection{Image Feature Extraction Based on Pattern Recognition Technology}

Due to the randomness of the image and the large amount of data, the difficulty of selecting an effective image feature in the image is increased, and the performance of the image recognition system is directly affected. Therefore, the first task to complete image recognition is to extract effective image features. However, in many practical problems, it is not easy to find the required features, or because they cannot be tested because of the conditional constraints [3]. Therefore, the complexity of feature selection and extraction tasks becomes one of the difficult tasks in building a pattern recognition system. The original characteristics or attributes of an image are called image features. Some of them are natural features and some are human features. Feature extraction is the process of extracting features that are filtered or transformed until they yield valid features. Its fundamental task is to select effective features and apply corresponding techniques for feature extraction. The result of image feature extraction based on pattern recognition technology is to give features that distinguish a specific image from other images.

In syntactic pattern recognition, the key issue is to infer the appropriate grammar using sample sets belonging to different pattern classes. The question of grammar inference is a primary issue. This method is based on basic assumptions, each pattern at least one grammar description. The identification and extraction of the grammar descriptions for each pattern form the core problem of designing a comprehensive pattern classifier [3]. The grammar inference problem involves using the sample pattern set in the study to obtain grammar algorithm development. So this can be seen as a method of learning using a limited and growing set of training patterns. In grammar pattern classification, strings belonging to a special pattern class can be used as sentences that form a language, and these sentences correspond to pattern classes. If each string belongs to the pattern class, the machine can identify the pattern class. For any string that is not in the pattern class, the machine decides whether it is a member of the language either rejects it or accepts it permanently. For automaton's automated sentences, accepting strings belongs to the special pattern class, and there are already some mature technologies.

\subsection{Pattern Recognition Based Image Recognition}

Image recognition is an advanced stage of image processing. Its research is to analyze and identify the visual images of surrounding objects through the instrument, so that effective conclusion can be obtained [4]. However, in order for computer systems to be able to recognize images recognized by the human visual system as well, one must develop computational methods and analyze image features, so that pattern recognition techniques are applied to image recognition, and then image features can be mathematically represented and taught. Computers can also recognize and identify these features.

Artificial neural networks have been widely used in image segmentation and object classification problems. These networks are essentially learning networks for the classification of pixels or objects in a scene. They are a collection of interconnected neurons that perform learning tasks in parallel. Neurons are modeled by biological neurons so they are named neural networks [4]. Depending on the type of learning process, these networks can be divided into supervised or unsupervised networks.

\section{Extraction of Electronic Information Features Based on Pattern Recognition Technology}

\subsection{The Concept and Content of Electronic Information Technology}

Electronic information technology refers to theories, techniques, and methods for acquiring, storing, transforming, transmitting, processing, and utilizing information through the use of electronic science and technology to realize the goal of information science and technology. It is mainly used for research, development, design, production, maintenance, and management of electronic devices. Information products and systems have the features of smart intensification, high efficiency, and network digitization [4]. From a technical point of view, the main contents of electronic information technology research are: information acquisition - information transmission information processing - information storage - information utilization. 1) Information acquisition 
includes the acquisition of voice, image, and physical parameters, as well as remote sensing technology, radar technology, and GPS positioning. 2) Information transmission. Include coding, modulation, detection, and fixed communications, mobile communications, and optical fiber transmission. 3) Information processing. Include voice, images, text, signals and other processing, and neural networks, pattern recognition. 4) Information storage. Include magnetic storage, optical storage, semiconductor storage, network storage. 5) Information utilization. Include control, display, information services, and network information retrieval.

\subsection{Application of Electronic Information Feature Extraction in Agricultural Production}

In agricultural production, organic integration of electronic information technology with basic disciplines such as agronomy and soil science can realize real-time monitoring and regulation of crops, soil, and the environment in the agricultural production process, as well as phenomena in agricultural production. The process was simulated to make rational use of agricultural resources, to save costs and improve efficiency, and to improve the ecological environment [5].

1) Information monitoring for example, real-time monitoring of environmental information in agricultural cultivation. Through the installation of environmental wireless data acquisition system, the sensor collects the planting region environment data (such as ambient temperature, soil temperature, ambient humidity and light intensity and other parameters), transmits it to the data collector, and then transmits it to the network database through the GPRS wireless data transmission module [5]. Through the Internet, agricultural production management personnel can refer to the real-time data of current changes in environmental parameters to provide a basis for rational agricultural production decisions.

2) Intelligent Control for example, automatic control systems are used in the greenhouse. Through the automatic monitoring of light, temperature, water, gas, and nutrient supply in greenhouses, according to the growth requirements of greenhouse plants, automatic control of window control, coiled membrane, circulating fan, temperature increase and decrease facilities, fill lighting, irrigation, fertilization and other environmental control equipment, adjust the environmental parameters in the greenhouse to reach the appropriate range, provide the best environment for plant growth, and achieve high automation in production and management [5].

\subsection{Application of Image Processing in Agricultural Machinery}

Agriculture-based mechanization led by science and technology is the basis of modern agricultural development. Intelligent agricultural machinery and equipment technology is the key to improving production conditions, increasing production efficiency, reducing production costs, and enhancing comprehensive production capacity [6]. The use of electronic information technology to develop advanced agricultural machinery and equipment suitable for the characteristics of China's agriculture, improve agricultural machinery and equipment information collection, intelligent decision-making and precise operation capabilities, to achieve intelligent control, operation automation.

1) Agricultural Machinery Manufacturing for example, digital design and flexible manufacturing technologies are used in the production of agricultural machinery and equipment. Using computer-aided design software, functions such as conceptual design, geometric modeling, engineering drawing generation, finite element analysis, optimization design, virtual assembly, kinematics simulation, machining method selection, process route formulation, and machining simulation testing can be implemented for agricultural machinery equipment. Reach the goal of improving design efficiency, improving design quality, reducing product development costs, shortening design cycles, and improving information management [6]. The use of flexible manufacturing technology to complete modern agricultural machinery and equipment manufacturing can improve manufacturing precision; achieve the most provincial investment, the highest production efficiency and the best economic efficiency.

2) Intelligent control of agricultural machinery most modern agricultural machinery and equipment use electronic technology control system, which can realize intelligent network control. For example: remote control sprinkler using communication technology, autopilot tractor, etc.; seeder, fertilizer applicator, sprayer, combine harvester, etc. using GPS positioning; farm 
maintenance management using computer technology, tractor onboard computer, etc.; use of automatic control technology's large-scale tractor job control system; the use of wireless video technology in the field operation of the operating locomotive monitoring device; the use of Beidou GNSS satellite high-precision positioning technology and vehicle automatic driving technology combined with agricultural machinery and equipment automatic navigation system [6].

\section{Summary}

Pattern recognition is a discipline that combines theory and practice. The way of teaching and learning is worthy of our research and exploration. It can also be seen that pattern recognition has considerable prospects, and the application of pattern recognition is supported by more and more people, and its effectiveness is also very significant. Therefore, it has also received the attention of various countries. It is precisely because each unit collectively pays attention to the pattern recognition of artificial intelligence. Pattern recognition technology can rapidly develop the application of electronic information feature extraction.

\section{Acknowledgement}

Fund Project: This paper is supported by the Foundation for Science and Technology Key Projects (as a Project of High and New Technology) of Henan Provincial Department of Science and Technology, 2017. Project No: 1722102210414.

\section{References}

[1] Sh. J. Wang, Theory and Application of New Model Recognition Model, Chinese Journal of Electronics, 2003, vol.1, pp.1-3.

[2] Y.W. Fan, Research and application of digital image processing, Journal of Zhejiang University, 2003, vol. 6, pp. 34-37.

[3] F.L. Qi, Pattern Recognition and Image Processing, Shanghai: Shanghai Science and Technology Press, pp. 11-13.

[4] W P.F. Application of Pattern Recognition in Electronic Information, Hohhot: Inner Mongolia University Press, 2010, vol. 9, pp. 16 - 18.

[5] J.H. Wang, Pattern Recognition Feature Extraction, Higher Education Press, 2013, pp. 43-47.

[6] Z.T. Zhu, Application of Pattern Recognition in Image Feature Extraction, Shanghai East China Normal University Press, 2011, vol. 8, pp. 18-21. 